\title{
Modern Trends in the Development of Audiovisual Media as Translators of Cultural Values
}

\section{Shesterina Alla}

Voronezh State University, Voronezh, Russia

shesterina8@gmail.com

\begin{abstract}
The relevance of the study is determined by the processes of active transformation of the audiovisual media sector and the transition to new forms of interaction with the audience. These changes affect, first of all, the sphere of translation of value cultural codes. The article analyzes both traditional and modern approaches to the work of media in the cultural and educational aspect. The author identifies constant components of audiovisual works (metaprograms, archetypal plots, traditional formats) which provide constant distribution of information about basic values. Modern trends in the development of the audiovisual media sector and their role in the process of broadcasting cultural values are analyzed.
\end{abstract}

Keywords: audiovisual media, broadcasting, cultural values, media communication, TV content

\section{Introduction}

The active mediatization of the social and cultural sphere becomes more and more evident along with the development of a technological component of the media system and the global digitalization of the society. Audio-visual production technologies, which were in many ways inaccessible to an ordinary person, are now becoming generally available, and almost every one of us can become a creator of video content and, therefore, a translator of collective and individual cultural codes. That is why today it is especially important to study the processes taking place in the system of audiovisual media and outline the key trends in their development.

The problem of fixing and spreading cultural values through audiovisual communication channels has been considered by researchers for a long time. So, even at the dawn of the formation of cinematography, scientists tried to outline the circle of those cultural codes that it conveyed. Even then it became clear that in this area there can be both the highest achievements and 
falls (for example, already in the middle of the twentieth century, researchers started talking about the threats associated with the broadcast of aggression and scenes of violence in films).

Such an ambiguous, positive-negative effect of audiovisual media related to the sphere of culture persisted later, when researchers were able to analyze the work of TV channels [Ilchenko and Okner, 2005; Bovshik, 2018]. It was noticed that along with programs promoting the spread of high cultural values, the media sector was actively developing, contributing to the so-called "down selection" [Dondurei, 2007].

The stratification in the audiovisual sphere is even more noticeable in relation to the newest media. Video blogging initially positioned itself as something different from TV. It detached itself from traditional formats, violating the canons of genres and norms of journalistic ethics. Its opposition was demonstrated in everything - from form up to content. Such scientists as E. L. Vartanova [Vartanova, 2018], A. G. Kachkaeva, S. A. Shomova [Kachkaeva, Shomova, 2017], D. A. Kirillov, T. A. Zaitseva [Kirillov, Zaitseva, 2017], S. V. Pobudey [Pobudey, 2017] and others studied the peculiarities of cultural values reflection in this sphere.

Despite the long history of understanding the potential of audiovisual media as translators of cultural codes, we have to admit the fact that there are still no trends that are constantly demonstrated in video production, and changing formats that meet the needs of a particular period in the development of society. In our study, we will try to fill this gap.

\subsection{Resource materials}

The research material consists of the most popular television programs and video blogs, which we will consider both in retrospect and from the standpoint of our own time. The empirical base of the study consists of broadcasting on Channel 1, the channel Rossiia, NTV, as well as the most popular video blogs in the network. The object of our research is, first of all, video content on cultural issues. The chronological period of the study is 2018-2020. However, to study the issue in retrospect, we will turn to earlier examples of audiovisual media.

\subsection{Methodology and study methods}

The goal is to identify constant forms of representation of cultural codes that are demonstrated at all stages of the development of audiovisual media, and modern trends in recent years. The analysis of texts has to accomplish two key tasks - the identification of constant features and the establishment 
of modern trends from the point of spreading cultural values. Comparative typological, historical and functional, textual methods and method of content analysis were used.

\section{Study description}

During the analysis of audiovisual content, we considered it important to grab attention to the constants we identified in order to determine which components of traditional and modern media ensure constant transition of social cultural codes from generation to generation. We concretized the active functioning in the visual media sector of such important components as metaprograms, archetypal stories and traditional formats, and examined them from the standpoint of participation in the process of disseminating basic values in the cultural sphere.

In our research, metaprograms mean certain angles of perception and description of an event, situation, process or problem that belong to a person (author or viewer). Researchers tend to identify several main metaprograms: people, values, process, past, achievement. If the author has a dominance of any metaprogram, he will describe the subject of his material in a certain way. Thus, one and the same event can be described through its participants' characteristics, feelings, experiences (metaprogram of "people"), through the sequence of actions that are taking place (metaprogram of "process"), through losses and gains, norms and their violations (metaprogram of "values"), through the causes and background of what is happening (metaprogram of "past"), through the results or consequences (metaprogram of "achievement"). The audience, perceiving a particular media text, can also highlight certain aspects in it, focusing on its dominant metaprograms. This forms the topical and genre preferences of the audience. Thus, a person whose perception is dominated by the metaprogram of "people" prefers personified forms of presenting information (conversation, commentary, essay, etc.). The viewer with the dominant metaprogram of "process" will choose dynamic forms (reportage, stream, game, etc.). It is important to note that metaprograms have kept their meaning in both traditional and new media over a fairly long period of time and each of them serves to broadcast those moral and cultural values that are transferred from generation to generation.

The archetypal plot means the principles of plot construction, collected from human experience and reflecting fundamental ideas about the world, universally reproduced in different cultures and eras. This interpretation, 
of course, is based on the concept of archetypes by K. G. Jung [Jung, 2019], as well as the theory of archetypal, universal plots by V. Ya. Proppa [Proppa, 1969], H.L. Borges [Borges, 1992], K. Booker [Booker, 2008], J. Polty [Polty, 1924]. From our point of view, archetypal plots are interesting because, regardless of technological breakthroughs, the emergence of new formats and even types of media, they continue to play the role of the basis of the narrative and broadcast cultural values, linking generations with common topics, problems and issues. It is archetypal plots that make it possible to actualize basic human values (life, health, warm social contacts) and basic human illusions (immortality, eternal love and friendship, faith in justice).

As for the interpretation of traditional formats, in this part of the study we relied on such a category as a frame, understood by us as a kind of structural basis for transmission, independent of its content.

The analysis of modern trends in the development of the audiovisual sector was carried out based on ideas about the peculiarities of the information behavior of the modern audience. Among its features, researchers name:

- replacing the user archetype by the creator archetype. The desire of the audience to actively interact with the video content (use of editing work to change the source material, commenting on the video or commenting with the help of video, etc.);

- scanning perception, leading to a reduction in video consumption time (using scrolling, accelerated, fragmentary or background viewing);

- parallel perception, work with several windows at the same time. This type of perception dictates the need to constantly hold the audience's attention;

- active appeal to amateur, non-professional recordings (the latter are often included in the structure of professional video;

- infantilism demonstrated by a tendency to watch game formats, hyperemotionality, instant emotionality, uncritical perception;

- voyeurism, a tendency to peep, which is realized by using a reportage camera, a hidden camera [Kachkaeva, Shomova, 2017].

Taking into account the peculiarities of the information behavior of the modern audience, we analyzed audiovisual media and identified basic, constant forms and modern trends in the development of this media sector and determined the nature of their participation in the broadcast of cultural codes. 


\section{Results and discussion}

\subsection{Constant components of audiovisual media}

After analyzing metaprograms as a significant component of the structure of the media text, we came to the conclusion that in matters of preserving and developing culture, the metaprogram of "values" plays a dominant role, transmitting values of both the spiritual and material plan. It is widely demonstrated in video works of cultural and educational topics. These are documentaries ("Frida na fone Fridy" [Frida at the background of Frida]), documentary series ("Krasuysya grad Petrov!" [Flaunt the city of Petrov!]), documentary TV shows ("Most nad bezdnoy" [The Bridge over the abyss]). In the material and everyday aspects, the metaprogram of "values" broadcasts certain norms to the audience or offers recipes ("Modnyy prigovor" [Fashion sentence],"Shkola remonta" [School of repair], "Chas suda" [Hour of the court]). We see this metaprogram at work in the latest audiovisual media as well. So, in the video blogging system, it is often used in such genres as guide, review, life hack, life style and others (for example, our author's blog "Konfliktologiya" [Conflict studies], broadcasting information about the value of open sincere communication).

The same can be said for the rest of the metaprograms. All of them, regardless of the changes taking place in society, continue to return the viewer to the essential issues by means of both traditional and the latest media. Thus, the metaprogram of "process" appears on television in reportage and dynamic game forms ("Neputevyye zametki" [Unlucky notes] or "Fort Boyard") and on the Internet in genres such as taste, prank, stream or story. It snatches out the current symbolic facts of reality and, from the totality of all broadcast phenomena, forms current trends in various spheres of people's lives (for example, it can determine fashion in the area of clothes, leisure time, moral standards, choice of profession, etc.). The metaprogram of "past" has an aim to keep information about the most significant achievements of mankind in the field of culture. Many cultural and educational programs on television are devoted to the same aim ("Moy serebryanyy shar" [My silver ball], "Kumiry" [Idols]) and in the Internet these are professional video blogs (the blog "OP-POP-ART" by Natalia Postrigai). The metaprogram of "achievements" transmits the peak forms of the human spirit. In traditional media, these are stories about someone's victories, about scientific discoveries, inventions ("Ochevidnoye-neveroyatnoye" [Obvious-Incredible]), and in the video blogging system, these are numerous tops and hates on the opposite, built, 
for example, on exposing pseudo-scientific information or on criticism of cultural experiments of low quality. Both traditional and modern media activate the metaprogram of "achievements" in stories about contests, festivals, games ("Golos" [The Voice], broadcasting of the Oscars, etc.).

The metaprogram of "people" deserves special attention and has recently become noticeably active in various types of media. We correlate this fact with an increase in the flow of information and the complication of the process of selecting relevant information. It is easier for the viewer to trust a specific person and receive information from him than to search for information of interest in various sources. People and not channels become the transmitters of values. That is why personal projects ("Pozner" or "The fate of a man with Boris Korchevnikov" etc.) are particular popular both on television and in the network media.

It is important, however, to emphasize that the dominance of a particular metaprogram is not a necessary factor in the success of a video work. On the contrary, the most successful video formats are those in which all or many of the metaprograms are activated (for example, "Genius Places" by Peter Weill). In this case, each viewer will find something of their own in the program or blog. An approach based on a combination of different metaprograms allows the author to expand his audience and appeal to everyone, broadcasting certain cultural values. The repetition of metaprograms from year to year, from era to era can be considered a constant sign of media development, preserving the key frames of information presentation.

The same can be said about archetypal subjects. Whatever changes take place in society, these stories are repeated from century to century in all areas of art and media forms. Let's consider the role of key archetypal subjects in the broadcast of cultural values.

One of the private plots of this kind is the "Feat of Prometheus", built on the hero's struggle for justice against an enemy superior in strength (this is why the plot has another name - "The fight against the titan"). The enemy can be a specific person, a group of people (for example, a political party), a dominant ideology. The hero of such works is a fighter who defends certain life principles. It conveys to the audience the belief in justice, the importance of fighting for what you think is right, adherence to principles. This is a person who asserts norms and principles for evaluating events. On the TV screen, we find such a plot in stories about heroes' feats (for example, in stories about the Great Patriotic War), in reports aimed at combating injustice ("Spetsial'nyy 
korrespondent" [Special Correspondent], "Svoya Pravda" [Personal Truth]). And on the Internet - this is the basis of revelations (for example - the blogs of Alexey Navalny, Alexander Nevzorov, the EVG channel) or an attempt to restore the historical truth (a number of headings "Parthenon" by Leonid Parfenov). It is difficult to overestimate the significance of this plot for the Internet. Video blogging was initially perceived by the authors as a platform for free and even oppositional speech. What could not be expressed on the screen, bloggers expressed on network platforms. The harsh tone of video blogs, the use of obscene vocabulary, and an amateur filming format are excused by this fact. The authors of the video blogs seemed to emphasize: "We broadcast different values, we are not like the hosts of traditional programs."

Another common archetypal plot on the web is the "Cinderella archetype." The essence of the plot is that a person deserving of reward and justice finally gets them with outside help. An example of the implementation of such a plot on TV is the "Chas suda" [Hour of the court] program, reporting on the solution of social and everyday problems (often in the housing sector). In a network platforms, we often find examples of the embodiment of such a plot in the lifestyle genre (blogs by Katya Klep or Sasha Spielberg, etc.) or in professional blogs where authors help the viewer to solve any problems (for example, the psychological blogs by Sasha Ivanov or Andrey Kurpatov).

Another important archetypal plot in the context of our research is "the systematization of philosophical categories". Its purpose is actualization and rethinking of deep questions for a person. On the TV screen, the programs "Gordon" and "Shkola zlosloviya" [School of Scandal] were a vivid demonstration of such a plot. It has been included in genres such as essays and comments. Moreover, this process is noticeable both in traditional and in the latest media. For instance. on the Internet, a similar story serves as the basis for many educational blogs (Arthur Sharifov's blog, MentalTV, SnailKick, Riddle, Mediapsikhologiya [Mediapsychology]). This type of plot allows you to discover something new in already known things, to return to topics that are repeatedly discussed by society at different stages of its development.

The broadcast of cultural values also takes place with the help of other archetypal plots - "rock", "cheating", "adventure", "sleeping beauty", "sudden disaster". These plots are experiencing some modification, but inevitably form the basis of both traditional and network video formats. The archetypal nature of the plots allows the audience to build associative links with already known realities, update background knowledge, and consolidate the learned values. 
A similar role is played by traditional television formats or frames for providing news, broadcasting information. The main reproduced frames include interviews, studio conversations, news stories, talk shows or commentary. They overlap with the main genres of journalism, are reproduced in both traditional and modern media, and can be filled with any content. So, a news story can include in various combinations such elements as synchronization, life, voiceover with video, stand-up. Other elements may also be present. Their combination may differ from plot to plot, and yet there will be few options for combinations, they will be repeatable. The audience, who perceives the stories every day, due to their repetition, can begin to trust the frame itself, regardless of the information included. Therefore, there is a risk in using traditional formats associated with the implementation of fake elements into the narrative (for example, the mocumentari genre, imitating the documentary frame well known to the audience).

In general, the problem of exploitation of traditional formats, archetypal subjects and metaprograms is urgent. By virtue of their repetition, recognition, familiarity, they can cause excessive trust in the audience and uncritical perception and lead to the grasping of inaccurate information. Authors of fake news rely on such mechanisms. It is no coincidence that most researchers define fake news as false reports imitating the authentic or as messages with reduced or absent informational value for the audience imitating valuable ones. This is a kind of imitation of reality which forms a media reality that is far from the first reality. And it is the established forms of information transmission that make their existence possible.

This is probably one of the reasons that dispose the authors of media works to search for new forms of communication with the audience.

\subsection{Modern trends in the development of audiovisual media}

First of all, it is necessary to note the quantitative growth of video content - not only on traditional platforms (such as Youtube, Yandex video or TikTok), but also on platforms that were previously focused on text or static illustrative information (Facebook, VKontakte). So, already in 2018, the director of ecology of the social network Vkontakte Sergey Paranko, in his speech at the annual scientific and practical conference "Journalism-2017", emphasized that it is video consumption that is the dominant form of information behavior in the social network. He also said that the stories captured by the users themselves are of the greatest interest to the audience. 
Large volumes of video can cause concern due to the fact that high-quality content broadcasting cultural value constants may be lost in the stream of low-quality video that conveys information of a dubious nature. It is no coincidence that researchers now record the presence of a significant number of fake markers in modern audiovisual media. Among the most common are:

- the use of video frames that are not related to the described event (the frames were filmed in a different place, the frames were filmed at a different time);

- the inclusion of video frames shot from a certain angle, which changes the assessment of an ongoing event (for example, a small event can be shown without general plans, which will create the illusion of a large number of people);

- videos with incredibly successful stunts, dangerous actions of characters or extremely painful blows (as a rule, they are filmed using sophisticated technologies, but they form the feeling of reportage and reliable information);

- changing the sequence of questions and answers in the interview, which leads to a change in meaning;

- use of announcements in the news release that distort the essence of the plot;

- whipping up emotions in credits, etc.

All these markers of fakes allow us to say that cultural values in such materials are broadcast in a distorted form and, if a fake video is discovered, trust can be undermined not only to the video itself, but also to the subject of display.

It is important, however, to note that a number of fakes have no negative basis. Moreover, in terms of broadcasting cultural values, they can be useful, since they appeal to such qualities of the audience as infantilism and a tendency to gamble. Attention in this sense deserves the use of deepfake technology. This is a technology that allows you to replace some parts of the video image with others without losing a sense of the reality of what is happening. For example, one person's face can be replaced by another person's face. In this case, the facial expressions of the new character will exactly duplicate the facial expressions of the real hero. Thus, it is possible to show any person doing stuff that he did not actually do. This image synthesis technique is based on the use of artificial intelligence and gives a very realistic result. Today it is mostly harmless and used in entertainment, but it can also be used for disinformation purposes. For example, deepfake technology was used at the Salvador Dali Museum in Florida where a special exhibition, Dalí 
Lives was organized to celebrate the artist's 115th birthday. The exhibition used a prototype of the artist generated by artificial intelligence, who communicated with visitors of the museum, shared stories of his paintings with them, and also took selfies and sent photos to visitors' mails.

Another example is a video in which Elon Musk sings the song "Trava $u$ doma” [Grass by the House]. During the video editing, the face of Elon Musk was put over the face of the guitarist of the Zemlyane group Igor Romanov. The video appeared on the Internet on May 31, 2020 on the day of the first launch of SpaceX spacecraft.

The use of deepfake technology is just one example of the development of such a trend as information gamification. The game format for presenting information is becoming more and more popular today and is included in the structure of television broadcasts of the information, analytical and documentary-artistic type. It has long been talked about its implementation in the format of infotainment (playing out news). One of the striking examples of the use of information on Russian television is in the films of Leonid Parfenov, in which the author plays up facts, presents them through the prism of theatre, imitation and even kitsch (for example, the film "Ptitsa Gogol" [Bird Gogol] made for the anniversary of the writer Nikolai Gogol). Today, infotainment is also used in TV programs ("Tsentral'noye televideniye" [Central Television]).

Elements of gamification are present in almost all genres of video blogging. They are more frequent in the sketch show ("This is horosho"), pranks and navels. They are present in a significant amount in other genres too. The activation of game elements supports another trend - the strengthening of the means of activating attention. This trend is associated with the multichannel and the possibility of simultaneous perception of information in several windows. First of all, it should be noted here more active work with submodalities - those are such parameters of the text that provokes a viewer's attention involuntarily when they suddenly change.

In the audiovisual sector, media submodalities can be divided into two types - visual and auditory. The visual submodalities with which modern media carry out active experiments include: stereo or mono; words or sounds; volume (loud or quiet); tone (sharp or soft); timbre (color of sound); the location of the sound source (far or near); duration (short or long sound); continuity or discontinuity; pace (faster or slower than usual); distinctness (distinct or muted).

The most common visual submodalities are associated (see with my own eyes) or dissociated (see myself in a picture). This submodality is often used 
by video bloggers in the form of shooting without a tripod, a non-standard shooting angle; color or black and white; framed or borderless; depth (flat or volumetric); location (e.g. right or left, top or bottom); distance (the picture is far or near you); brightness; contrast; sharpness (blurry or focused); mobility (film or slide); speed (faster or slower than usual); quantity (split screen or composite image); the size.

By modeling these submodalities we can draw the audience's attention to content that might otherwise seem boring to the viewer. It is easy to see that the activation of submodalities is becoming a trend of the network platforms, and then it moves to television screens, allowing the viewer's attention to be fixed on the values being broadcast.

Finally, the personification of information is becoming another trend in the development of modern audiovisual media. Above, we offered an explanation of the reasons for this phenomenon. Let's look at it in more detail.

Researchers today note the dominance of the so-called open subjectivity in the media sphere. Reanimation of subjectivity leads to the fact that "my opinion" is presented as a priority for society. This process, which is objectively developing in the media, can have its pros and cons. On the one hand, if the author of the statement has a rich, interesting, deep and developed personality, then he can broadcast the values of the "vertical plan", encouraging a viewer for self-development and self-creation. On the other hand, a shocking author of a video work can go down to a very low level of broadcasting values and thereby significantly lower the cultural level of his audience. The situation is aggravated by the rejection of external control in the modern media. The ethical norms of each individual become the only criterion for his behavior. The ability to hide one's real life personality allows one to demonstrate qualities of character suppressed by the morals of society. This is reinforced by the prevalence of heuristics over evidence. Evidence assumes a set of facts arranged in a certain order dictated by logic, where one clearly follows from the other. However, such a linear scheme may not be available to the author, not interesting to the audience and is impossible in the conditions of constant returns and leaps that characterize modern media consumption.

\section{Conclusions}

All of the abovementioned things allow us to say that modern media in the aspect of broadcasting cultural values play a double role: on the one hand, they preserve basic knowledge and broadcast it to the audience, using 
both traditional and the latest formats and providing a sense of stability in the post-truth era. On the other hand, they can significantly lower the general cultural background of the audience - not only in the area of experiment with the form of presenting the message, but also in the conditions of using traditional approaches. It is important for the authors of modern media texts dealing with cultural issues to rely on archetypal plots, fixed formats, classical metaprograms. But at the same time it is very important not to use them in order to introduce controversial, unverified, questionable information. Of course, it is important for them to search for new forms, taking into account the transformation of the information needs of the audience, but at the same time it is important to remember the effect of "down selection" and not sacrifice values for the sake of momentary fame.

\section{References:}

1. S. N. Ilchenko, O.A. Okner. Television in the internet age. Saint-Petersburg (2005).

2. B. A. Bovshik. Information agenda: Media reality filtered through TV. Idei i novatsii, 2 (2018), pp 9-13.

3. D. Dondurei. Censorship of reality. Television: Directing Reality. Moscow (2007), pp. 10-18.

4. E. L. Vartanova. On the basic concepts of the "digital media field" in Russian media research. Medialmanach, 2 (85) (2018), pp. 8-16.

5. Mul'timediynaya zhurnalistika [Multimedia journalism] / Ed. A. G. Kachkayevoy, S. A. Shomovoy. Higher School of Economics Publishing House, Moscow (2017).

6. D. A. Kirillov, T. A. Zaytseva. Video blog as a communication channel. Alley of Science, 5 (2017), pp. 42-47.

7. S. V. Pobudey. Video blog as a means of formation of historical knowledge. Belarusian State University. Scientific conference of students and postgraduates. Proceedings of the 74th scientific conference of students and postgraduates of Belarusian State University: in 3 parts. Part 3. Minsk (2017). pp. 294-298.

8. K. G. Jung. Psychological types. Moscow (2019).

9. V. Ja. Propp. Morphology of a fairy tale. Moscow (1969).

10. H. L. Borges. Collection: Short Stories. Essay. Poems. Saint-Petersburg (1992).

11. Ch. Booker. The seven basic plots. Why we tell stories. NY (2008).

12. G. Polti. The thirty-six dramatic situations. Franklin (1924). 